\title{
GUARANTEEING QoS IN BLUETOOTH SCATTERNET SENSOR NETWORK
}

\author{
R. Dhaya ${ }^{1}$, V. Sadasivam ${ }^{2}$ and R. Kanthavel ${ }^{3}$ \\ ${ }^{I}$ Department of Information Technology, National Engineering College, Tamil Nadu, India \\ E-mail: dhayavel@yahoo.co.in \\ ${ }^{2}$ Department of Computer Science and Engineering, Manonmaniam Sundaranar University, Tamil Nadu, India \\ ${ }^{3}$ Department of Computer Science and Engineering, Government College of Engineering, Tamil Nadu, India \\ E-mail: kanthavel@yahoo.com
}

\begin{abstract}
Bluetooth is a low power and cost-effective short range wireless network technology working in $2.4 \mathrm{GHz}$ ISM band. Bluetooth can be implemented either in piconet or Scatternet. In piconet the devices can communicate with each other forming a network with maximum of 8 nodes (1 master and 7 slaves). Two or more piconets can be connected through a common Bluetooth device (a gateway or bridge) to form a Scatternet. But the difficult thing is to achieve Quality of service $(Q o S)$ in Bluetooth particularly in the Scatternet. Quality of service refers to the efficient management of system resources, which includes the parameters like bandwidth, delay, jitter etc. This paper addresses guaranteed QoS in a Bluetooth scatternet by considering mainly two constraints namely Packet Loss and Waiting time .The above two constraints are met in a Bluetooth scatternet under high traffic congestion, which increases packet delay and causes channel wastage thereby affecting QoS. In the existing First In First Out (FIFO) scheme the slaves are served by the master in accordance with the arrival of the request offered by respective slaves in the form of 'first come first serve' technique. So a proposal to simulate a new priority based scheduling scheme like DST (Dynamic Scheduling Technique) has been implemented, which overcomes the pitfalls like packet loss, waiting time, and high congestion in the FIFO scheduling scheme. The results shows that the proposed DST scheduling that carries dynamic priorities for the requesting slaves by employing dynamic scheduling technique, which assures and guaranties the QoS parameters.
\end{abstract}

Keywords:

Bluetooth, Sensor Network, Scatternet, Quality of Service, Dynamic Scheduling

\section{INTRODUCTION}

Bluetooth is a standard for short range, low power, and low cost wireless communication that uses radio technology with the transmission rate up to 1 Mbps. The Bluetooth channel is divided into time slots of $625 \mathrm{~s}$ length, where a different hop frequency is used for each slot. It operates in the $2.4 \mathrm{GHz}$ spectrum and uses a frequency hopping technique to reduce interference from other devices operating in the same band. The Bluetooth network plays a vital role in the data communication between devices in wireless Personal Area Network (PAN).The data exchange between the Bluetooth devices must be reliable, efficient and secure within the estimated timeliness and these features can be achieved by guaranteeing the QoS parameters in the concerned Bluetooth network [1]. As far as a single user system is concerned, the phenomenon of request-response is achieved without any interruption and hence the quality of service is retained. As the number of users get increased in the system like distributed system, we switch over to the new technology called Bluetooth, which is implemented either by piconet or scatternet system.
Due to multiple requests from many devices connected in a Bluetooth network occurring at the same time, network overhead results. For any data transmission based network reliability, accuracy, completeness, consistency, and robustness must be achieved. There are several scheduling schemes previously proposed namely FIFO, Round Robin Scheduling etc which resulted in several scheduling and resource allocation problems. Some of the major pitfalls encountered in these schemes that affect the quality of service in Bluetooth scatternet are depicted below in terms of Packet loss, over delay, Miss Probabilities and Traffic congestion [10].

\subsection{BLUETOOTH NETWORK CONFIGURATION}

Piconet: A cluster of up to eight Bluetooth devices, one device holds the role of master, while the rest of the devices are slaves. Maximum of seven slaves can be active in a piconet at any given point in time, as shown in Fig.1(a). Any Bluetooth device can function within a piconet as a master, a slave or a bridge. These roles are temporary and exist only as long as the piconet itself exists. The master device selects the frequency, the frequency-hopping sequence, the timing that means, when the hops will actually occur and the polling order of the slaves. The master is also responsible for instructing the slave devices to switch to different device states for periods of inactivity [8] . A master and slave must exchange address and clock information in order for the slave to join the master's piconet. Bluetooth devices have a unique Global ID used to create a hopping pattern. The master radio shares its Global ID and clock offset with each slave in its piconet, providing the offset into the hopping pattern. A slave must be able to recreate the frequencyhopping sequence of the piconet it has joined, must know which frequency to use at which time, and must synchronize itself with the master's clock [2].

A Bluetooth bridge device or gateway interconnects two or more piconets for multi-hop communication. The bridge communicates with all the piconets connected to it by aligning itself with the clocking of each piconet when it is ready to communicate. A bridge device may be a slave in all of the piconets to which it is connected, or it may be a master in one piconet and a slave in the others.

Scatternet: Two piconets can be connected through a common Bluetooth device (a gateway or bridge) to form a scatternet, as shown in the Fig.1 (b). These enable devices which are not directly communicating with each other, or which are out of range of another device, to exchange data through several hops in the scatternet [7].

To overcome the link wastage problem in Bluetooth scatternet scheduling, several algorithms have recently been 
proposed. Although they can provide all of the links of a bridge node with fair service opportunities but they may cause waste of wireless resources since different Bluetooth devices may have various traffic characteristics. This paper deals with the problems discovered in the existing Scheduling scheme and suggest solutions to resolve the problems.
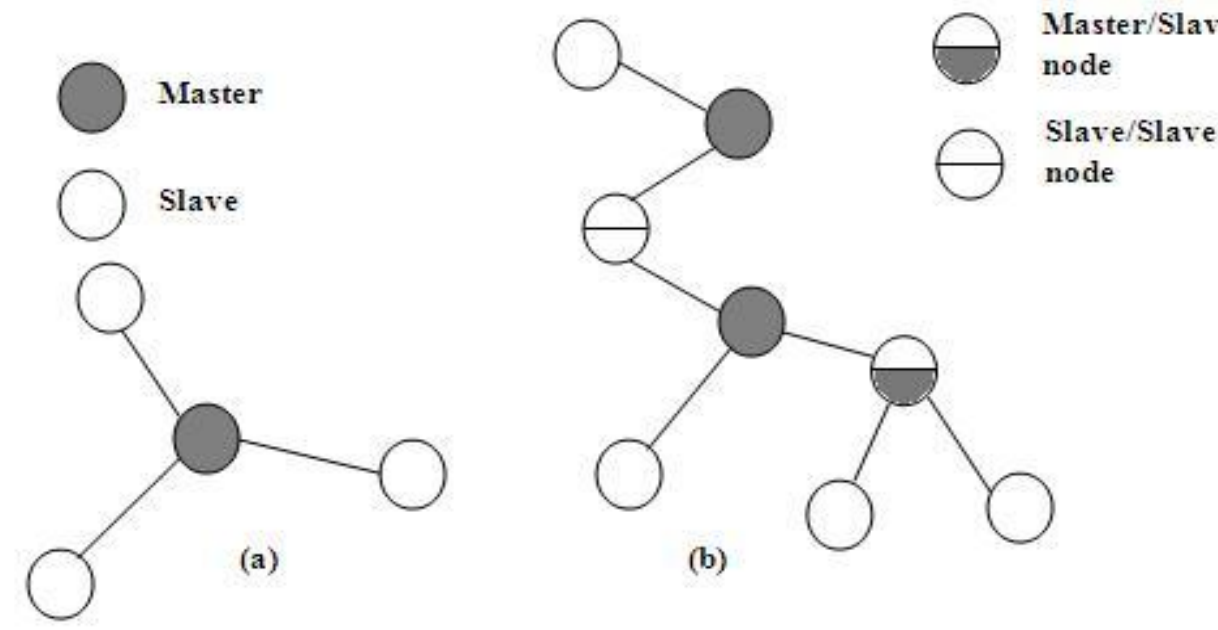

Fig.1. (a) Piconet, (b) Scatternet

\section{PROPOSED SOLUTIONS OF DST}

To improve the quality of service in the Bluetooth scatternet using the dynamic scheduling technique the following modules have been done.

- Formation of Bluetooth scatternet.

- Designing DST scheduling scheme.

- Performance comparison between FIFO and DST.

\subsection{FORMATION OF BLUETOOTH SCATTERNET}

Each device in a Bluetooth network is termed as a node. The Bluetooth specification defines the Bluetooth nodes which grouping themselves for the purpose of communication. Bluetooth scatternet system is formed by connecting two or more piconets through a common device called gateway or bridge which is a slave or master in the respective piconets. The proposed scatternet comprises two piconets and each carries 8 nodes shown in Fig. 2.

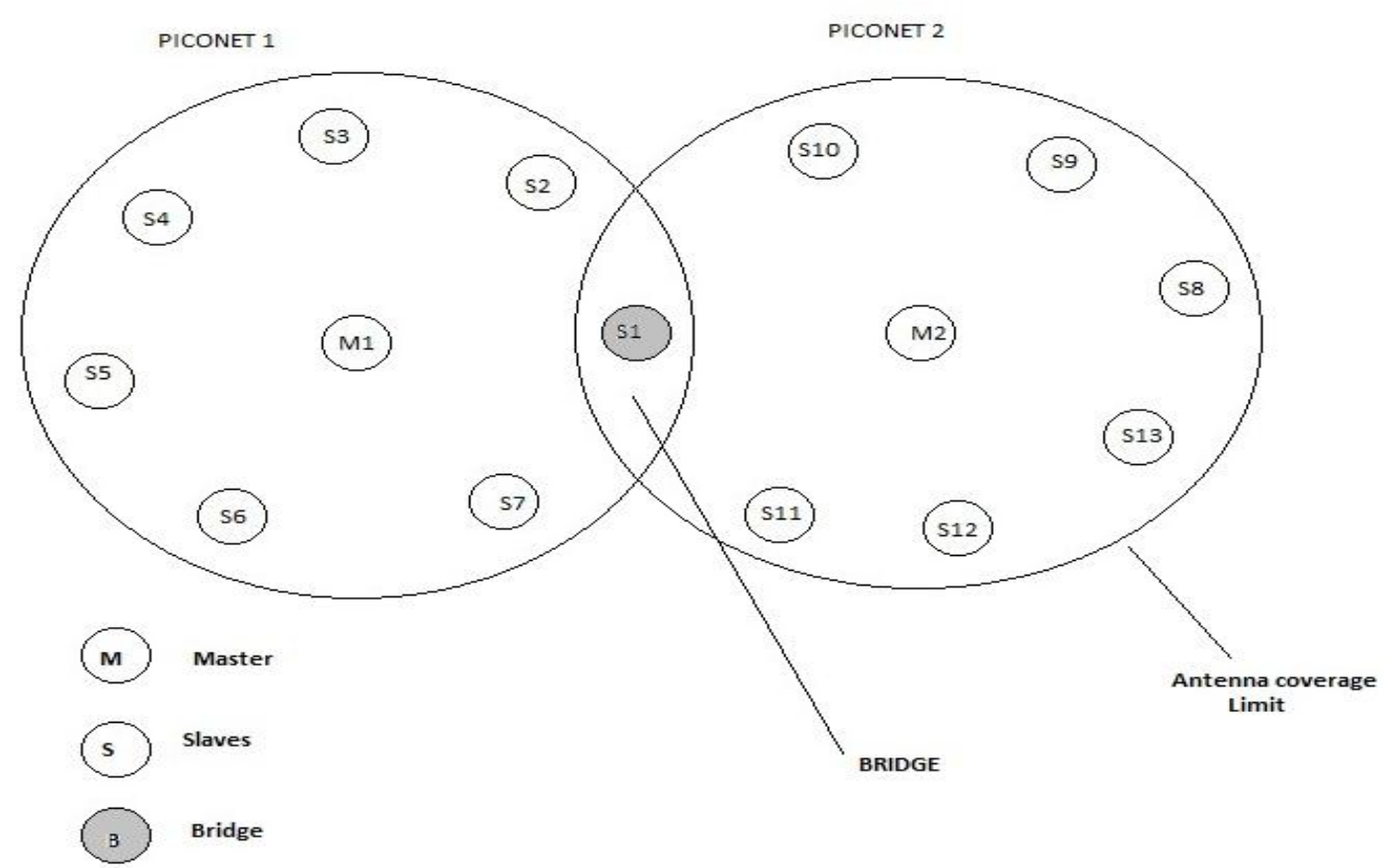

Fig.2. Proposed Structure of Bluetooth scatternet 
However, when a piconet is formed between two or more devices, one device is dynamically elected to take the role of 'master', and all other devices assume a 'slave' role for synchronization reasons. Piconets have a 3-bit address space, which limits the maximum size of a piconet to 8 devices $\left(2^{3}=\right.$ 8), i.e. 1 master and 7 slaves. The devices participating in both piconets can relay data between members of both ad-hoc networks. Using this approach, it is possible to join together numerous piconets into a large scatternet, and to expand the physical size of the network beyond Bluetooth's limited range [16]. In an intra piconet system the QoS parameters like delay, bandwidth, jitter etc which are not affected to a large extent, as the data transmission limited within a boundary. But in inter piconet system the Qos parameters are highly affected as the data has to be transferred among several piconets are hence over complexity results [3]

Each slave node in the Bluetooth scatternet must be located with in the antenna coverage established by the respective master. If the devices are not within the coverage area data communication is entirely collapsed. Hence this is one of the prominent criteria which must be satisfied by all the slaves in each piconet to achieve the efficient communication. If a device wants to participate in more than one piconet, it has to synchronize to the hopping sequence of the piconet it wants to take part in. If a device acts as a slave in one piconet, it simply starts to synchronize with the hopping sequence of the piconet it wants to join. After synchronization, it acts as a slave in this piconet and no longer participates in its former piconet [6].To enable synchronization a slave has to know the identity of the master that determines the hopping sequence of a piconet. Bluetooth network assures a well defined secure data transmission between the nodes. Each node in a network has a unique IP address for its own identification in the network [5].

\subsection{DESIGNING DST SCHEDULING SCHEME}

This technique makes use of priority levels of the requesting slaves and hence forms a new packet queue based on the packet size of the requesting slaves. In the existing scheduling scheme of FCFS (First Come First Serve), there is no difference in small sized packet request and large sized packet request and whichever request comes first is given data which affects the QoS. But in the proposed DSS (Dynamic Scheduling Technique) the slaves are served by suitable priorities for the requesting slaves [13].

The packet size of the requests are sorted from slaves to be served on the basis of the packet size in the form of increasing order .If the packet size of two or more are identical, their respective waiting time are calculated and served on the basis of the increasing order of waiting time of slaves. By using this scheme only the large sized request must wait for a long time and the small sized requests are served before the turn for large sized request, irrespective of the arrival sequence of the requests to the master [9]. In a basic Bluetooth network called piconet the normal scheduling schemes itself acceptable because the routing path needs to trace just a single master only, where high interruptions are negligible [14], [15]. But in the case of Bluetooth scatternet the best scheduling schemes must be adopted to achieve QoS and routing scheme is one of the important factors affecting the QoS to a large extent. Routing is defined as the selection of the shortest path to be traversed from the requesting device to the destined device [4]. Dynamic scheduling refers to the allocation of suitable priorities for the requesting slaves at the run time. The following factors are used in the design of DST algorithm [10].

$$
\begin{aligned}
& \lambda \text { - arrival rate of packets } \\
& r \text { - Priority of slaves } \\
& q \text { - Packet queue } \\
& \text { w - Waiting time } \\
& \text { p - Packet size }
\end{aligned}
$$

Let $\mathrm{P} 1$ be the first piconet and P2 be the second piconet which are inter connected through the slave1 of the piconet P1 which acts as the bridge B and hence a link between P1 and P2 is established. The number of connections to be made must be predefined by the user in terms of the number of slaves . The algorithm needs to distinguish among inter piconet scheduling (among the piconets) and intra piconet scheduling (within a single piconet). When the data transmission is within a piconet, the packet needs to be transferred from the requesting slave to the serving slave through the corresponding master. When the data transmission is among the piconets the concerned packet must be transferred from the requesting piconet to its master followed by the bridge and master of the serving piconet and finally the destined slave. Fig.3 illustrates the communication flow of DST algorithm.

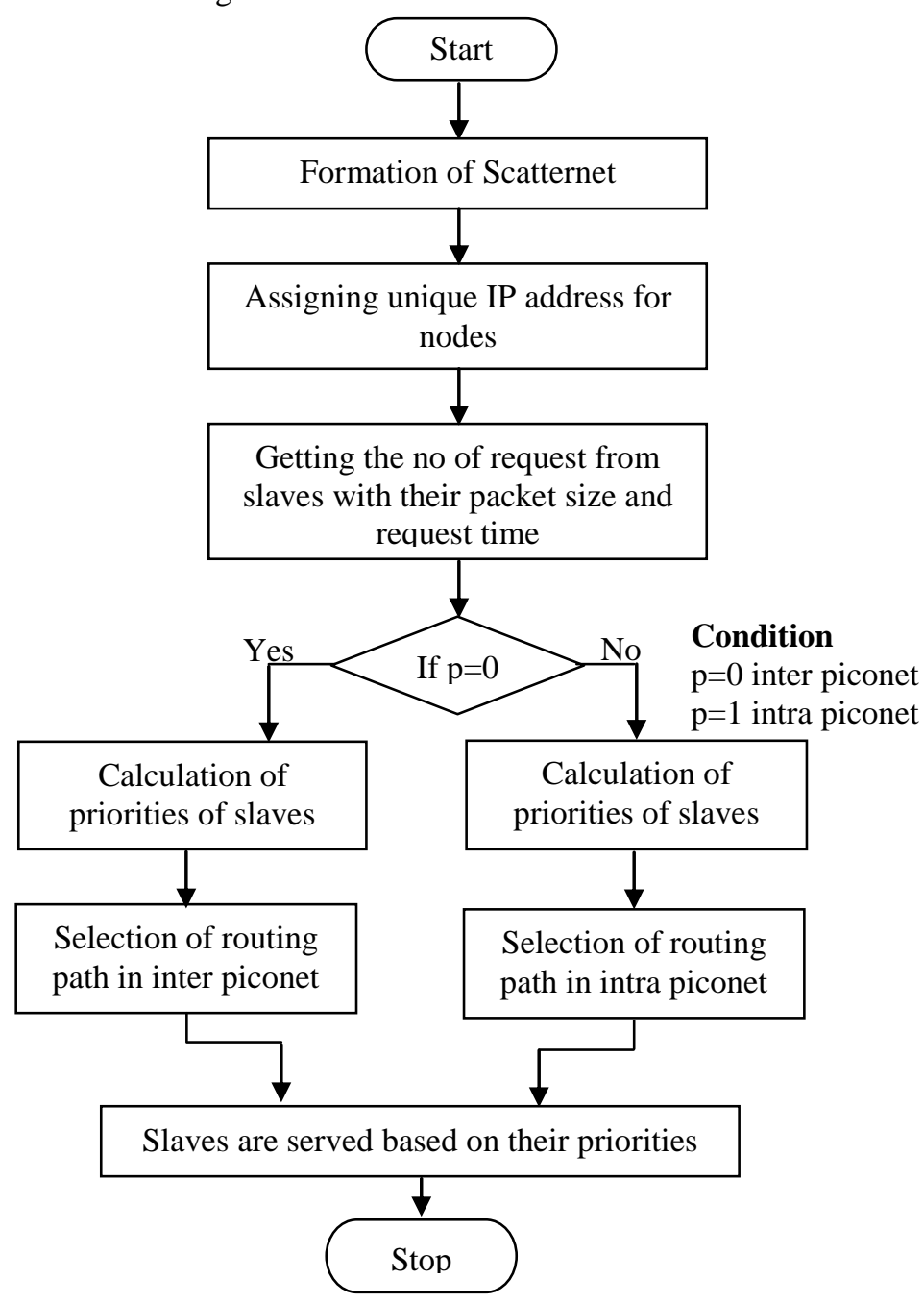

Fig.3. Communication flow for DST algorithm 
Consider there are two requests in the system. One is within a piconet and the other one is among two piconets .Under this condition intra piconet scheduling gets prioritized over the inter piconet scheduling and hence intra request is served first before inter request. The cases based on the packet size are explained below.

\section{CASE 1}

S1: Requested to M1 at time $\mathrm{t} 1$.

S2: Requested to M1 at time $\mathrm{t} 2$.

$\mathrm{Ps}_{1}$ : Packet size of $\mathrm{S} 1$.

$\mathrm{Ps}_{2}$ : Packet size of S2.

\section{CASE 2}

$\mathrm{S} 1:$ Requested to M1 at time $\mathrm{t} 1$.

S2: Requested to $\mathrm{M} 1$ at time $\mathrm{t} 2$.

$\mathrm{Ps}_{1}$ : Packet size of $\mathrm{S} 1$.

$\mathrm{Ps}_{2}$ : Packet size of S2.

$\mathrm{Wt}_{1}$ : waiting time of $\mathrm{S} 1$.

$\mathrm{Wt}_{2}$ : waiting time of $\mathrm{S} 2$.

\section{CASE 3}

$\mathrm{S} 1$ : Requested to M1 at time $\mathrm{t} 1$.

S2: Requested to M2 at time $\mathrm{t} 2$.

$\mathrm{Ps}_{1}$ : Packet size of $\mathrm{S} 1$.

$\mathrm{Ps}_{2}$ : Packet size of $\mathrm{S} 2$.

$\mathrm{Wt}_{1}$ : waiting time of $\mathrm{S} 1$.

$\mathrm{Wt}_{2}$ : waiting time of $\mathrm{S} 2$.

When there are two requests to be handled, one is within a piconet and another is among two piconets then the network meets two situations, based on packet size and waiting time. Under this condition even if one third of packet size of S1 is less than half of the packet size of S2 and one third of waiting time of $\mathrm{S} 1$ is greater than half of the waiting time of $\mathrm{S} 2$, and $\mathrm{S} 1$ is served first before $\mathrm{S} 2$.

The data transmission rate for Bluetooth based devices ranges up to $1 \mathrm{Mbps}$ which is equivalent to $122 \mathrm{kbps}$.

For example:

Bluetooth speed / sec is 1000000 bits. Let the packet size (bits) is 3500000 . So the total time needed for transmission is $3.5 \mathrm{sec}$.

The particular packet must be transferred between the respective devices with in the deadline period. In the above example the packet transmission must be finished successfully within the deadline of $3.5 \mathrm{sec}$.

\subsection{PERFORMANCE COMPARISON BETWEEN FIFO AND DST}

Scheduling refers to the efficient utilization of system resources along with the peripherals to complete the requests offered by the slaves. The scheduling scheme must be selected in accordance with the requirements so that the network throughput is achieved in a manner as expected by the user $[9,10]$. The selected scheduling scheme must be cost effective and time efficient.

Let us consider a piconet shown in Fig.4, with master labeled as $\mathrm{M}$ and the slaves are S1, S2, S3, S4, S5, S6. Let three slaves in the network namely S1,S2,S5 at the time instances $\mathrm{t} 1, \mathrm{t} 2, \mathrm{t} 3$ with packet sizes $236512,2021,532$ respectively. These three slaves are served on the basis of FIFO pattern as follows.

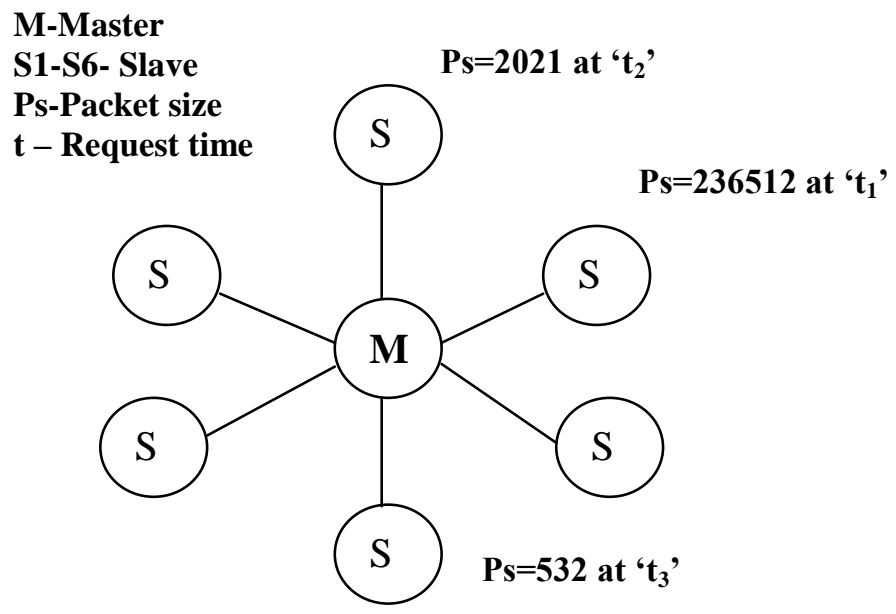

Fig.4. Representation of slave requests

Let us consider a piconet shown in Fig.4, with master labeled as $\mathrm{M}$ and the slaves are S1, S2, S3, S4, S5, S6. Let three slaves in the network namely $\mathrm{S} 1, \mathrm{~S} 2, \mathrm{~S} 5$ at the time instances $\mathrm{t} 1, \mathrm{t} 2, \mathrm{t} 3$ with packet sizes $236512,2021,532$ respectively. These three slaves are served on the basis of FIFO pattern as follows.

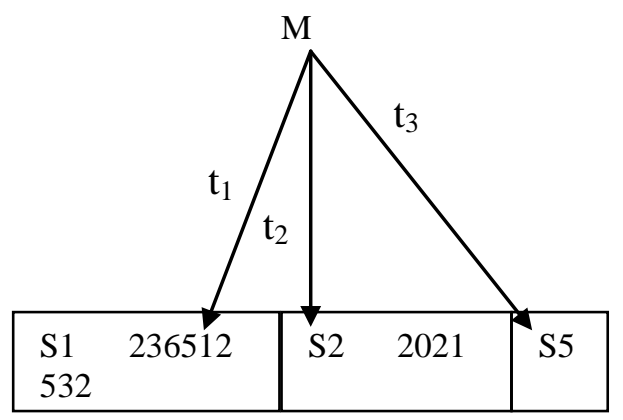

Fig.5. FIFO operations

The FIFO scheme works on the basis of first come first served pattern. From the Fig.5,it is obvious that the slave S1 with the packet size 236512 is served first as it had arrived the queue first .But the remaining two requests made by slaves S2 and S5 with packet sizes 2021 and 532 are served next to S1. In this scenario the slaves S2 and S5 with least packet sizes need to wait for a longer time until the slave S1 (arrived first) with large size request is served completely. Hence the slaves S2 and S5 must wait until S1 is served, even though their request are minimum sized compared to the first arrived slave S1 with maximum sized packet [11].

Under this FIFO technique, the Bluetooth system efficiency is highly affected and long time high traffic situation in encountered. As the minimum sized requests have to wait for a long time results delay degrading the quality of service parameters. 


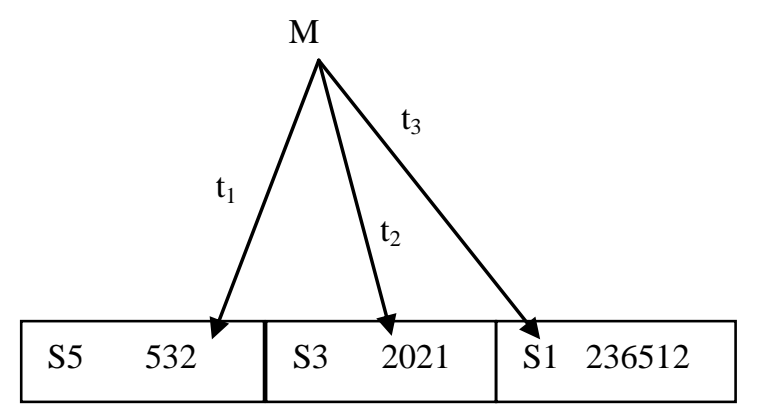

Fig.6. DST operation

Dynamic scheduling refers to the allocation of suitable priorities for the requesting slaves at the run time. Here priority is established on the basis of packet size and waiting time of slaves. The Fig. 6 depicts that the slave's request with minimum packet size is served first irrespective of the sequence of arrival of slaves request to the master.

The Packet size of slaves S3 and S5 are too small when compared to the packet size of S1.In DST scheduling scheme S5 and S3 are served completely before the scheduler offers the choice for S1 to get served. S5 and S3 do not have to wait for a long time until S1 is served and the traffic in the Bluetooth scatternet is highly reduced. From the above results it is clear that the DST scheme guaranties a better improvement on the Quality of Service parameter than the FIFO scheme in a Bluetooth scatternet [12].

\section{EXPERIMENTAL ENVIRONMENT}

The dynamic scheduling algorithm is implemented in network simulator (ns2) under MAC protocol. The experiments are conducted in a Bluetooth scatternet of two piconets linked through a bridge. The aim of the experiment is to enhance the quality of service in the Bluetooth scatternet during the data transmission effected in request-response fashion. The experiment performed with the transmission range of approximately $100 \mathrm{~m}$. In this experiment, the slaves are served on the basis of the priorities in terms of packet size and waiting time. In this DST scheme, the requested slaves with minimum sized packets are not subjected to wait for long time and hence the delay in network is reduced abundantly.

This section explains the simulation results and performance analysis of DST algorithm.

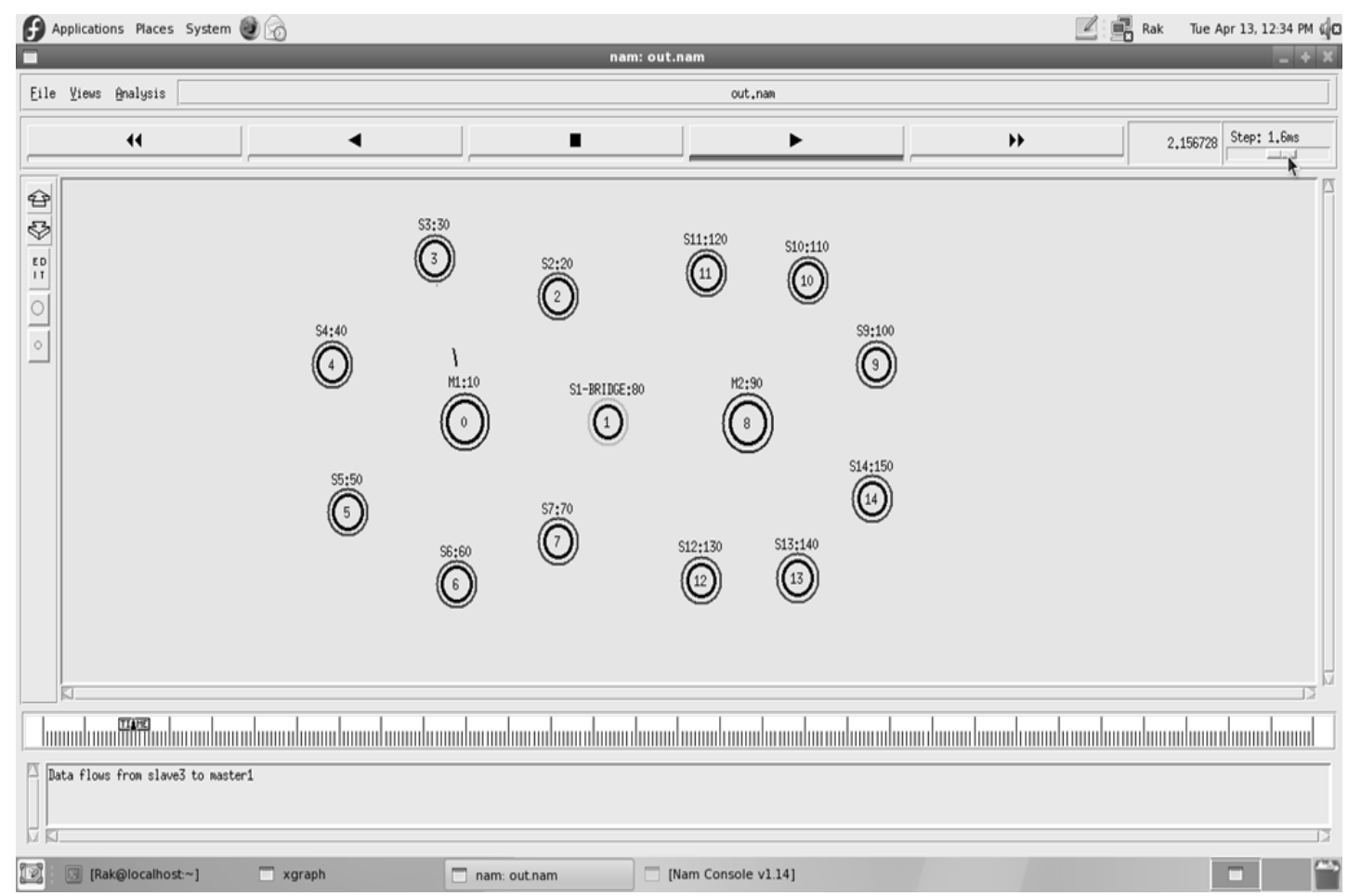

Fig.7. Intra piconet request

The Fig. 7 shows that the slave $\mathrm{S} 3$ of piconet P1 requests its master M1. This slave must wait until the master allocates the time slot for serving the requested slave. 


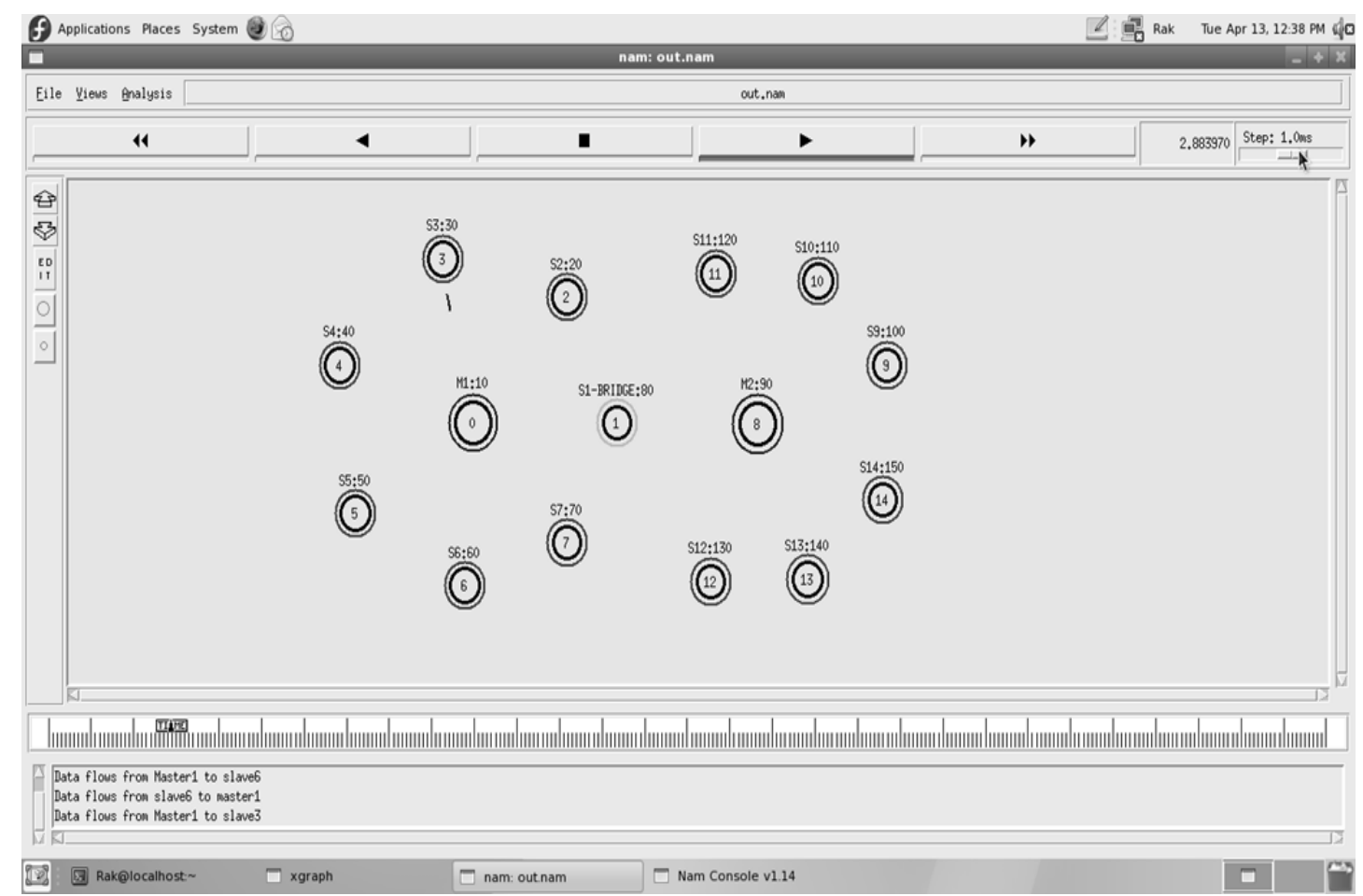

Fig.8. Intra piconet response

The Fig. 8 shows that the requested S3 is served by its master M1 when the time slot for S3 is recognized by the master M1.

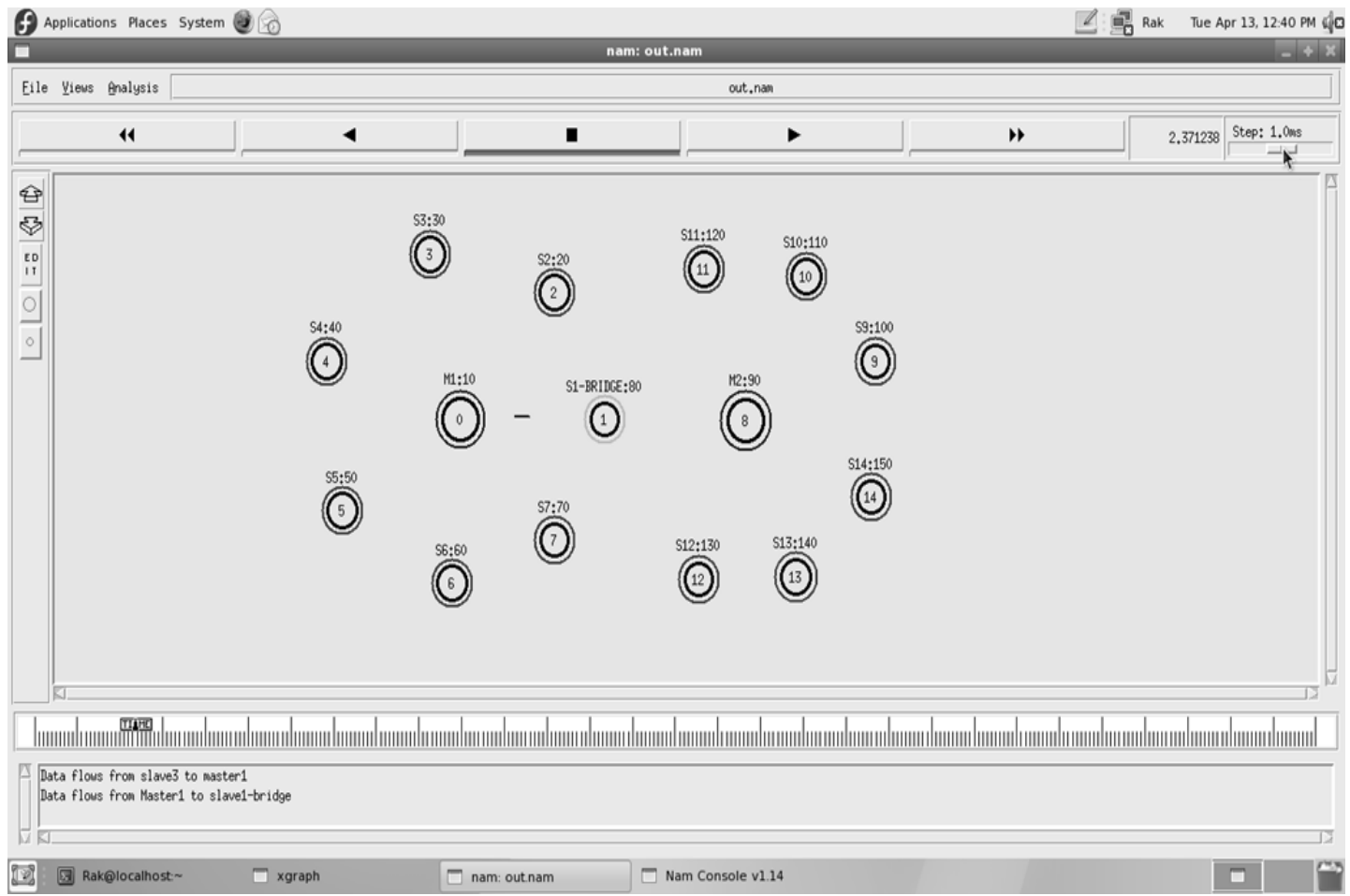

Fig.9. Inter piconet request 
The Fig. 9 shows that the slave S3 of piconet P1 needs the data from the slave $\mathrm{S} 10$ of piconet $\mathrm{P} 2$. This request path is from slave S3 of piconet P1 to its master M1 and master M2 of piconet $\mathrm{P} 2$ through a bridge node and finally to the destined slave $\mathrm{S} 10$ of piconet $\mathrm{P} 2$.

Likewise the slave $\mathrm{S} 10$ of piconet $\mathrm{P} 2$ sends the requested data to the slave S3 of piconet P1.The serving path from slave $\mathrm{S} 10$ of piconet $\mathrm{P} 2$ to its master is M2 and via master M1 before finally to the requested slave $\mathrm{S} 3$ of piconet $\mathrm{P} 1$.

The waiting time for the proposed DST and existing FCFS is compared. Let S1, S3, S6 be the three slaves requesting the master with packet sizes 4500125, 5900,750 Bytes respectively arriving in the sequences at $\mathrm{t} 1, \mathrm{t} 2, \mathrm{t} 3$. The waiting time of these three slaves to be served by the master are calculated and shown in the Table 1.

Table 1.Waiting time of slaves in FIFO and DST

\begin{tabular}{|c|c|c|}
\hline Slaves & $\begin{array}{c}\text { Waiting } \\
\text { time(FCFS)ms }\end{array}$ & $\begin{array}{c}\text { Waiting } \\
\text { time(DST)ms }\end{array}$ \\
\hline S1 & Null & 6.35 \\
\hline S2 & 4500 & 5.6 \\
\hline S3 & 4505.9 & Null \\
\hline
\end{tabular}

It is clear that the first requested device does not need to wait as it is in the head of the queue leads its waiting time is said to be null. At time $t 1$ the network encounters heavy traffic as the first request $\mathrm{S} 1$ has the maximum size among all the requests. Because of this delay in the network quality of service is heavily affected in the case of FCFS. It is obvious that the slave S6 which has the least sized request among all the available requests which is served by the master first. Comparing the two scheduling schemes, the following are studied.

The slave S3 must wait for $4500 \mathrm{~ms}$ in FIFO whereas in DST just $5.6 \mathrm{~ms}$ is only needed which is too low compare to the former technique.

- The slave S6 has to wait for $4505.6 \mathrm{~ms}$ in FIFO whereas in DST it does not need to wait as it is first served.

- Finally, in FIFO two slaves S3 and S6 are affected heavily whereas in FIFO just only one slave $\mathrm{S} 1$ is affected to a negligible level.

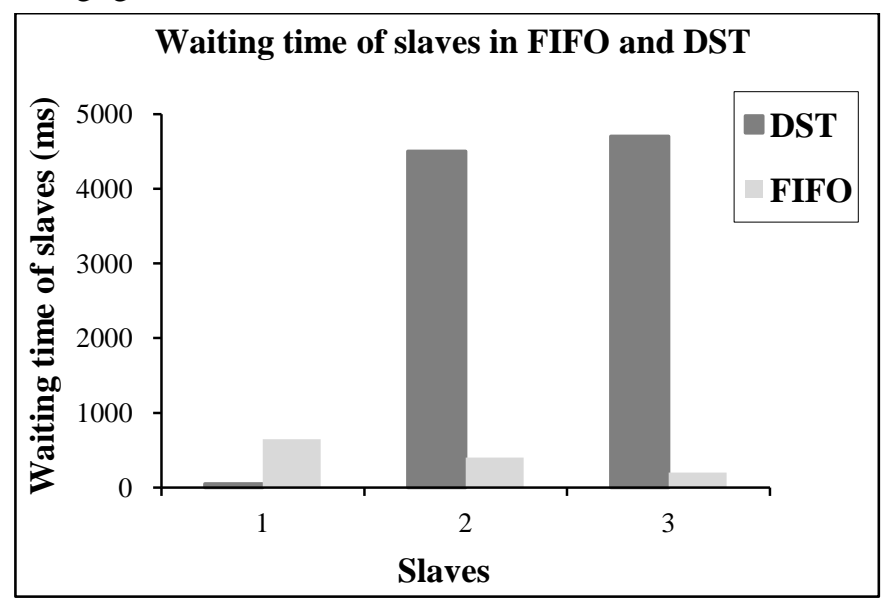

Fig.10. Graphical Comparison between FIFO and DST
From the Fig.10, it is confirmed that the priority based scheduling results in an enhanced performance compared with FCFS scheduling schemes. The time factor governs a major control on maintaining the quality of service in any network based system. Hence the response time must be minimized to the maximum level as possible which in turn automatically improves the quality of service. From the proposed DST Bluetooth channel is efficiently used with the achievement of commendable system throughput. Slaves with minimum sized packets are served first and Packet loss is minimized in terms of correct switching through the bridge. Thus the quality of service is guaranteed in Bluetooth scatternet by employing dynamic scheduling technique which is based on assigning suitable priorities of slaves.

\section{CONCLUSION}

To enhance the quality of service in Bluetooth scatternet a dynamic scheduling scheme (DST) has been implemented under three modules. The first module deals with the construction of scatternet with two piconets linked through a device called bridge. The second module includes the design of the DST in which antenna coverage for each device is established and the requesting slaves are served on the basis of two priorities namely packet size and waiting time. The final module explains the performance comparison between the previous FIFO scheme and the proposed dynamic scheduling technique. It is also clearly found that DST gives better results than FIFO in terms of packet size and waiting time. From the results it is clearly identified that the DST scheme shows better performance than FIFO in the scatternet network. Thus it is concluded that a priority based scheduling Bluetooth scatternet system guarantees QoS with better throughput compared to FIFO. From the DST scheme it is obvious that the probability for the packets with minimum sizes to be served is high. Apart from the techniques employed in priority based scheduling scheme of DST, the reduction in number of unnecessary piconet switching events may be treated as a valuable future scope which further improves the quality of service.

\section{REFERENCES}

[1] Adrian perrig, john stankovic, and david wagner, 2004,"Security for wireless sensor networks", Communications of the ACM, Vol. 47, No.4, pp 53-57.

[2] Chris Karlof and David Wagner, 2003, "Secure Routing in Wireless Sensor Networks: Attacks and Countermeasures",IEEE, pp. 113-127.

[3] Cordeiro, C., Abhyankar, S and Agrawal, D.P., 2006, "Sclable and QoS-Aware Dynamic Slot Assignment and Piconet Partitioning to Enhance the Performance of Bluetooth Ad Hoc Networks", IEEE Transactions on mobile Computing, Vol.5, No.10, pp. 1313-1330

[4] K. Saleem, N. Fisal, S. Hafizah, S. Kamilah, and R. A. Rashid, 2009,"Ant based Self-organized Routing Protocol for Wireless Sensor Networks", International Journal of Communication Networks and Information Security (IJCNIS), Vol.1, No.2, pp. 42-46. 
[5] K. Saleem1, N. Fisal, S. Hafizah, S. Kamilah, and R. A. Rashid, 2009," A Self-Optimized Multipath Routing Protocol for Wireless Sensor Networks", International Journal of Recent Trends in Engineering, Vol.2, No.1, pp.93-97.

[6] Konrad Iwanicki and Maarten van Steen, 2009, "On Hierarchical Routing in Wireless Sensor Networks" In Proc .IEEE, pp. 133-144.

[7] Laiali Almazaydeh, Eman Abdelfattah, Manal Al- Bzoor, and Amer Al- Rahayfeh, 2010,'Performance Evaluation of Routing Protocols in Wireless Sensor Networks", International Journal of Computer Science and Information Technology, Vol.2, No.2, pp. 64-73.

[8] Piyakul Tillapart, Pratit Santiprabhob, Sanguan Thammarojsakul and Thanachai Thumthawatworn, 2004, "An Approach to Hybrid Clustering and Routing in Wireless Sensor Networks", IEEEAC paper \#1319, Final Version, Updated, pp. 1-8.

[9] Shreedhar.M and G. Varghese, 1996, "Efficient fair queuing using deficit round-robin", IEEE/ACM Transaction on Networking, Vol.4, No.6, pp. 375-385.

[10] Stefano Basagni, Raffaele Bruno,Chiara Petrioli, 2003,"A Performance Comparison of Scatternet Formation Protocols for Networks of Bluetooth Devices", Proceedings of the First IEEE International Conference on Pervasive Computing and Communications, pp. 56-65.
[11] Tarique Haider and Mariam Yusuf, 2009,"A Fuzzy Approach to Energy Optimized Routing for Wireless Sensor Networks", The International Arab Journal of Information Technology, Vol.6, No.2, pp. 179-188.

[12] Vidhyapriya and Dr P T Vanathi, 2007," Energy Efficient Adaptive Multipath Routing for Wireless Sensor Networks", IAENG International Journal of Computer Science, Vol.34, No.1, pp. 24-31.

[13] Wang Yu and Zheng Guping, 2009, "Advance Detour Strategy for Geographic Routing in Wireless Sensor networks", In Proc,IEEE computer society , pp. 296-299.

[14] X.Y. LIE, I. Stojmenovic and Y. Wang, 2006,"Partial Delaunay Triangulation and Degree Limited Localized Bluetooth Multihop Scatternet Formation", IEEE Transactions on Parallel and Distributed, Systems, Vol.15, No.4, pp. $350-36$.

[15] Y. Wang, I. Stojmenovic and X.Y.LI, 2006,"Bluetooth Scatternet Formation for Single-hop Ad Hoc Networks Based on Virtual Positions", In Proc. 9th IEEE Symposium on Computers and Communications ISCC'2004, pp.156-159.

[16] Yang-Ick Joo, Tae-Jin Lee, Doo Seop Eom, Yeonwoo Lee and Kyun Hyon Tchah,2004, "Power-efficient and QoS-aware scheduling in Bluetooth scatternet for wireless PANs", IEEE Transactions on Consumer Electronics, Vol.49, No.4, pp. 1067-10. 